\title{
Luminescence in the Mineral Realm to Teach Basic Physics Concepts
}

\author{
Michele Lustrino $^{1,2}$ \\ ${ }^{1}$ Dipartimento di Scienze della Terra, Sapienza Università di Roma, P.le A. Moro, 5, 00185, Roma \\ ${ }^{2}$ Istituto di Geologia Ambientale e Geoingegneria - CNR c/o Dipartimento di Scienze della Terra, Sapienza \\ Università di Roma, P.le A. Moro, 5, 00185, Roma \\ Correspondence: Michele Lustrino, Dipartimento di Scienze della Terra, Sapienza Università di Roma, P.le A. \\ Moro, 5, 00185, Roma. E-mail: michele.lustrino@uniroma1.it
}

Received: December 28, 2020 Accepted: April 10, 2021 Online Published: April 30, 2021

doi:10.5539/jel.v10n3p83 URL: https://doi.org/10.5539/jel.v10n3p83

\begin{abstract}
Every substance is associated to emission of electromagnetic radiation whose peaks are essentially influenced by temperature. Hot bodies (i.e., at $\mathrm{T}>700{ }^{\circ} \mathrm{C}$ ) emit electromagnetic radiation in the field of visible light (incandescent light). The radiation emitted by cold bodies (i.e., at normal ambient conditions) in the visible light range is defined as luminescence. Luminescent light is emitted by exciting substances by means of electron fluxes (e.g., those generated in a cathode ray tube), using photon fluxes associated to electromagnetic radiations with wavelengths lower than $\sim 390 \mathrm{~nm}$ (fluorescence), by mechanical stress (triboluminescence), by small temperature increase (thermoluminescence) or by biological processes (bioluminescence).

The value of luminescence demonstrations in teaching is unique. Indeed, by illuminating specific minerals and other substances (such as chicken eggs, seashells, fossils, wood, scorpions, soda drinks) with non expensive ultraviolet (UV) lights, it is possible to introduce the audience to several scientific arguments. Among these, the most important are: a) the concept of radiation and electromagnetic spectrum, including the $\gamma$ - and x-ray as well as the radio wave extremes; b) the discovery and the essence of natural radioactivity; c) the concept itself of visible light and the nature of the colours; d) the adaptability of the human eyes to the environment; e) the principles of the atomic structure as well as the basic concepts of energy quantization, including the photoelectric effect; f) the basic aspects of black-body radiation.
\end{abstract}

Keywords: fluorescence, luminescence, mineralogy, electromagnetic spectrum, radioactivity, atomic structure

\section{Introduction}

Our eyes are blind to nearly the entire electromagnetic spectrum. With this term, we refer to the range of electromagnetic waves characterized by specific physical properties such as wavelength, frequency and photon energy. These three concepts are strictly linked so that, once defined one of them, it is possible to calculate the other two. The range of the electromagnetic spectrum comprises waves spread over more than twenty orders of magnitude in length, from less than one billionth of millimeter to more than several ten thousand kilometers. Radiation of electromagnetic waves represents a way to emit or transmit energy by means of mass-free particles known as photons. We are immersed in a bath of radiation. Radiation is around us and bombards us, and our life depends on it, albeit mostly inconsciously.

The great majority of the electromagnetic spectrum is invisible to our eyes, with the exception of a quite insignificant range defined as "visible light" (Figure 1). Our brain is able to process the signals that it receives after visible light impacts upon the retina, and we customarily associate specific wavelength ranges with the name of specific "colours". Contrary to popular thought, colours are not inherent properties of light, as are energy or wavelength, nor of the matter. Rather, colours are the subjective interpretation that is the consequence of energy causing chemical changes in retinal proteins. It is also true that matter is not associated to specific colours, these changing as consequence of body temperature and external influences (e.g., stresses or particle fluxes).

Light visible to human ranges from wavelengths close to $390 \mathrm{~nm}(\mathrm{~nm}=$ nanometer $=$ one billionth of a meter $)-$ a colour that we define as violet - to the longest visible wavelengths of $\sim 770 \mathrm{~nm}$ - with a colour that we define as red. In between these two extremes, there are intermediate wavelengths that we conventionally define as blue $(\sim 450 \mathrm{~nm})$, green $(\sim 550 \mathrm{~nm})$ or yellow $(\sim 600 \mathrm{~nm})$. Wavelengths can be transformed into frequencies according 
the relation:

$$
v=c / \lambda
$$

where $v$ (the "n" letter of the Greek alphabet) is the frequency, i.e., the number of times a wavelength reaches the same position (e.g., the crest) in one second, $c$ is the speed of light $(\sim 300.000 .000 \mathrm{~m} / \mathrm{s})$ and $\lambda$ is wavelength (in m; Figure 1).

Photon Energy

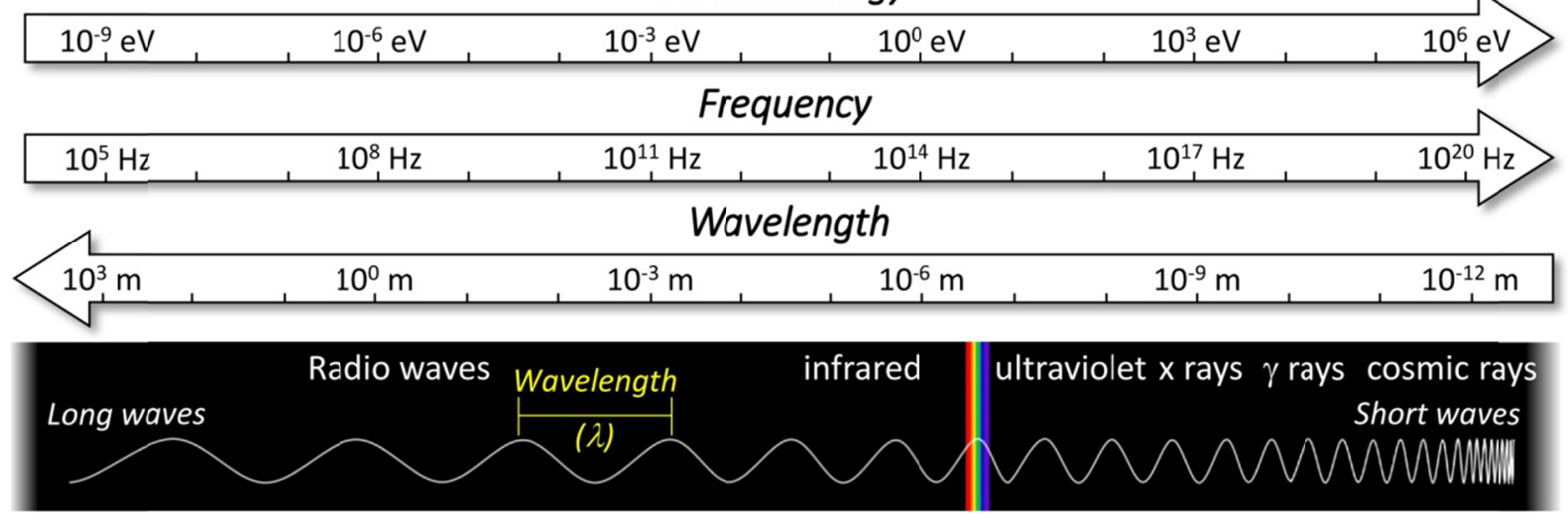

Figure 1. Electromagnetic spectrum showing the wavelength (in $\mathrm{m}$ ), the frequency (in $\mathrm{Hz}$ ) and the energy (in eV). The visible light occupy a very narrow range of the spectrum. Infra in Latin means "below". Infrared indicates the frequencies below red colour. Ultra in Latin means "over". Ultraviolet indicates the frequencies higher than violet colour.

As a comparison, it is possible to define the colours as discrete values of a continuous spectrum in the same way we define in western music the specific notes (or pitch) in the number of seven (plus five "middle notes" in between, for a total of twelve basic sounds or frequencies). A continuous range of wavelength (and frequency) characterizes the sound waves, and the notes played with a piano use only a very small number of fundamental frequencies. Differently from electromagnetic radiation, acoustic radiation is characterized by frequencies that are perceived as similar if grouped in a ratio equal to any integer power of two (e.g., 0.5, 2, 4 times, and so on). Consequently, the various pitches (or notes) are grouped in a number of basic classes that is multiplied several times. An example is the number of a piano frets, which continuously repeat the notes from A to G. The note "A" corresponds to a frequency of $440 \mathrm{~Hz}(78.4 \mathrm{~cm}$ acoustic wavelength) and the note $\mathrm{G}$ to a frequency of $\sim 1570 \mathrm{~Hz}$ $(22 \mathrm{~cm})$. Our ear can listen the fundamental frequencies in a relatively large range, from $20 \mathrm{~Hz}$ to $\sim 20 \mathrm{kHz}$ (mostly from 100 to $10 \mathrm{kHz}$ ). Sound waves with lower or higher frequencies are not audible for us. In other words, pitches (or notes) could be associated to a given acoustic frequency in the same way colours are associated to a given electromagnetic frequency (and, thus, a given wavelength).

What follows are the results of a three-year experience of the Museo Universitario di Scienze della Terra (MUST - Earth Sciences Museum of Sapienza University of Rome) with fluorescent minerals. The participation of more than ten thousand visitors to MUST during several programs and exihibits convinced us that fluorescent minerals are powerful tools to increase curiosity general people about not-easy-to-digest concepts like electromagnetic radiation, radioactivity, electronic orbitals and the origin of colours. This can be done using easily available and inexpensive tools such as rocks and minerals, plus wood chunks, scorpions, marine shells and chicken eggs. Of course, these concepts are presented at very elementary levels, to grab the attention of young people with the aim to inspire them to pursue studying scientific disciplines at secondary schools or at university levels. The "wow" effect of fluorescent minerals is a seductive method enabling us to reach these goals.

\section{Luminescence}

Luminescence is the ability of a matter to emit visible light as consequence of an energy input. Examples of energy input may be a temperature increase (thermoluminescence), mechanical stress (triboluminescence), biological reactions (bioluminescence), excitation by means of electron flux (cathodoluminescence) or energetic electromagnetic radiations (radioluminescence and fluorescence; Mazzoleni and Appiani, 2010). 
Phosphorescence is the emission of visible light for a time variable from a fraction of seconds to many days after energy input ends (a sort of afterglow). Tenebrescence is defined as the reversible persistence of a visible colour different from the original, after the end of the energy input, a phenomenon that can last also several days as visible under normal daylight.

Luminescence is a characteristic common of a few hundred of the nearly 5700 mineral species recognized by the International Mineralogical Association. Of these, less than 30 species account for greater than $95 \%$ of the classical minerals found in collectors' showcases. The most striking luminescent effects are by far those related to fluorescence and, to a minor degree, to phosphorescence. Many types of minerals show brilliant fluorescent colours under specific wavelengths (ultraviolet - UV - lights), while others are characterized by different fluorescent (and phosphorescent) colours under different wavelength UV lights.

\section{Types of UV Sources}

Custom, practicability and availability have determined the three main types of ultraviolet (UV) sources, defined as UVA, UVB and UVC lights. UVA (longwave ultraviolet) lights emit light with a wavelength close to $365 \mathrm{~nm}$ (range $\sim 315-400 \mathrm{~nm}$ ), UVB (midwave ultraviolet) lights peak around $300 \mathrm{~nm}$ (range $\sim 290-315 \mathrm{~nm}$ ) and UVC (shortwave ultraviolet) lights peak around $254 \mathrm{~nm}$ (range $\sim 200-290 \mathrm{~nm}$ ). UVA lights are known as Wood bulbs or black light bulbs, but now UVA flashlights equipped with nearly monochromatic LEDs are gaining popularity in the marketplace. The cost of UVA flashlights ranges from $\sim 2$ to 500 USD depending on the intensity of the light (expressed as $\mathrm{mW}$ or $\mathrm{mW} / \mathrm{m}^{2}$ ) and the width of the emitted spectrum, with the narrowest (more focused) being the most expensive. Several low-cost UVA flashlights claim to emit true $365 \mathrm{~nm}$ light, but, in reality, their peak is quite wide and centred around 395 rather than $365 \mathrm{~nm}$. Such cheap UVA lights are consequentially of limited use in the study of fluorescent minerals because much of the emitted wavelengths can be seen by human eyes, in the form of a violet colour. Strictly speaking, a true electromagnetic source focused at $365 \mathrm{~nm}$ should emit little or no visible light and effectively this is what is seen with professional UVA lights. More professional flashlights are equipped with a filter that selectively absorbs wavelengths longer than $\sim 385 \mathrm{~nm}$, i.e., those falling in the visible range of the electromagnetic spectrum. One such filter with a good quality/price ratio is commercially sold with the label ZWB2.

UVB lights are tubes, because UVB LEDs are still too expensive and have insufficient power output to be used in mineral displays. This wavelength is less commonly used by fluorescent mineral collectors, but can offer surprising results in specific minerals (e.g., calcite specimens from Terlingua, Texas, US) characterized by a peculiar fluorescent colour when observed with UVB light. These lights are used to treat diseases such as psoriasis, atopic dermatitis and vitiligo. In order to be used by fluorescent mineral collectors, visible light output must be blocked using specific filters, such as Hoya 325C or UL254S.

The sources for UVC lights are bulbs in which electrons excite mercury vapour, which then in turn emits $254 \mathrm{~nm}$ light in addition to visible light. A $254 \mathrm{~nm}$ bulb is not expensive (ranging from $\sim 5$ to $50 \mathrm{USD}$, depending on the power and the socket). What is expensive are the filters required to block visible light to much less than $10 \%$ wavelengths $>410 \mathrm{~nm}$. The cost of the filters depends on the efficiency with which it absorbs unwanted wavelengths, and the degree to which it is resistant to solarization. This latter is a phenomenon characterized by a reduction in the transmission of UVC light as result of its reaction with the filter glass, but the most recent filters seem to suffer this problem to a much lower degree. The cost of a practical UVC lamp ranges from $\sim 100$ to $>2000$ USD depending on the power and the filter size. Unfortunately, at the moment there are nearly no UVC light dealers in the entire Europe and the only market were to buy professional UVC lamps is US.

\section{Energy of Photons}

The energy of the photons emitted by any electromagnetic source is proportional to the frequency of that radiation, and inversely proportional to wavelength. This means that the shorter the wavelength the higher the frequency. The energy of a photon is characterized by its frequency divided by the Planck constant:

$$
\mathrm{E}=\mathrm{vk}
$$

where $E$ is energy expressed in Joules $(\mathrm{J}), v$ is frequency expressed in hertz (Hz; $1 /$ second) and $k$ is the Planck constant $\left(\sim 6.63 \times 10^{-34} \mathrm{Js}\right)$. For example, a light with a wavelength of $525 \mathrm{~nm}$ is associated to the colour green by our eyes. The frequency of this wavelength (eq. 1) is: $\left(3 \times 10^{8} \mathrm{~m} / \mathrm{s}\right) /\left(525 \times 10^{-9} \mathrm{~m}\right)=5.71 \times 10^{14} \mathrm{~Hz}(=1.75 \mathrm{x}$ $10^{-15} \mathrm{~s}$ ). The energy of such a photon is (eq. 2) $=5.71 \times 10^{14} 1 / \mathrm{s} * 6.62 \times 10^{-34} \mathrm{Js}=3.78 \times 10^{-19} \mathrm{~J}$.

\section{The Discovery of the "Invisible Light"}

The exact nature of the visible light has interested alchemists and scientists for centuries. In 1666, 23 years-old Isaac Newton used a triangular glassy prism to demonstrate that sun light was the sum of different rays (violet, 
indigo, blue, green, yellow, orange and red). Newton interpreted the different colours as very tiny particles, with size increasing from violet (the most refracted colour) to red (with the least refraction) because he thought that heavy particles were more difficult to deviate from the straight path. Few years after, the Dutch physicists Christian Huyghens proposed the ondulatory theory of the light, in contrast with the corpuscular theory of Newton. This is not the place to discuss these theories, but now it is known that both the interpretations are correct. Indeed, it is possible to interpret light as having a dual nature, at the same time as a mass-free energetic particle (photon) and as a wave, as mathematically demonstrated by the French physicist Luis de Broglie in his $1924 \mathrm{PhD}$ thesis.

In 1800, the German-borne British astronomer and physicists Wilhelm Herschel found an answer to the intriguing question of the ability of sun light to heat matter. Using a glassy prism, Herschel separated the colours of white light and placed sensitive thermometers in the different colour strips. He found that the temperature increased from violet $(\sim 390 \mathrm{~nm})$ to red $(\sim 770 \mathrm{~nm})$. With great surprise, he realized that the temperature continued to increase moving far away from the red in the invisible light range. Herschel discovered the existence of invisible light responsible for much of the heat of the sun (Herschel, 1800), later known as infrared radiation (Figure 1).

The year after, the German physicist Johann Ritter asked himself: "If there is a radiation beyond the red, why do not propose the existence of a radiation beyond violet?" Since alchemist times, it was well known that sun light had the power to darken some silver salt ( $\mathrm{AgCl}$; silver chloride). Ritter knew that violet had the greatest power to darken this substance. In 1801, he placed a thin layer of $\mathrm{AgCl}$ over a white paper strip under the sun light decomposed with a glass prism. Surprisingly, he found that the silver salt became darker and darker at wavelengths shorter than violet. He found the second source of invisible light, later known as ultraviolet light (Figure 1).

\section{Use of UV Lamps}

Ordinary fluorescent bulb lights (e.g., those used in shop signs or in work places) consist of a generator of ultraviolet energy. Inside the bulbs there is a coating of fluorescent powder or phosphor, which the ultraviolet causes to fluoresce brilliantly, thereby producing visible light. The UV lamps described here are different from classical fluorescent lamps, being simply emitters of radiant energy in the ultraviolet spectrum range. Their energy output in the UV range can generate the fluorescence in natural objects such as minerals and rocks.

Fluorescent colours of minerals have a special ability to grab the attention of undergraduate as well as well more advanced students and even expert adults. Fluorescent minerals actually emit light rather than reflecting incident light as other objects do. They often show vivid and very saturated colours and frequently nearly monochromatic wavelengths not commonly observed in other natural objects. Above all, the extraordinary effect of fluorescence serves to transform ugly chunks of rocks into spectacular stars in home or museum displays (Figure 2). What is needed is not expensive, considering the emotional payoff. A box with black painted walls with variable size and a Plexiglas (or polycarbonate) or glass screen (with a minimum thickness of 2-3 mm) in front is basic. The screen is essential for two reasons: 1) it prevents dust accumulation and 2) it stops the dangerous radiation from UVC lamps. Different types of UV sources can be used, but considering that the most spectacular results are those obtained using UVC lamps.

\section{How Are Fluorescent Colours Generated?}

The electrons orbiting around the nuclei of the elements move extremely fast (in the order of thousand of $\mathrm{km} / \mathrm{s}$, which is in the order of one hundredth the velocity of light, still an incredibly high speed). The exact position of a given electron in a single moment cannot be specified, as explained by the uncertainty principle proposed by the 26-year-old German physicists Werner Heisenberg in 1927. Electrons move in specific volumes at a given range of distances from the nucleus. These volumes are known as orbitals and can be described using the first two quantic numbers in the dynamics of a quantum system such as the atom. The first (principal quantum number $n$ ) indicates the distance from the nucleus, while the second (azimuthal quantum number $l$ ) indicates the shape of the orbital.

Fluorescent minerals, on the other hand, behave in a partially different way, because they release back only a part of the excitation energy. Indeed, the excited electrons (of fluorescent minerals) release a small amount of the excitation energy as heat. This means that when the electron relaxes from its excited state to the ground level it emits only a portion of the original photon energy (Figure 3). The difference between input and output energy is called the Stokes shift, after George Stokes, who first described this process to explain the colour change in the alkaloid quinine sulphate $\left(\mathrm{C}_{40} \mathrm{H}_{50} \mathrm{~N}_{4} \mathrm{O}_{8} \mathrm{~S}\right)$ and the green-to-blue colour change of some natural fluorites $\left(\mathrm{CaF}_{2}\right)$ from Alston Moor (UK; Stokes, 1852). 

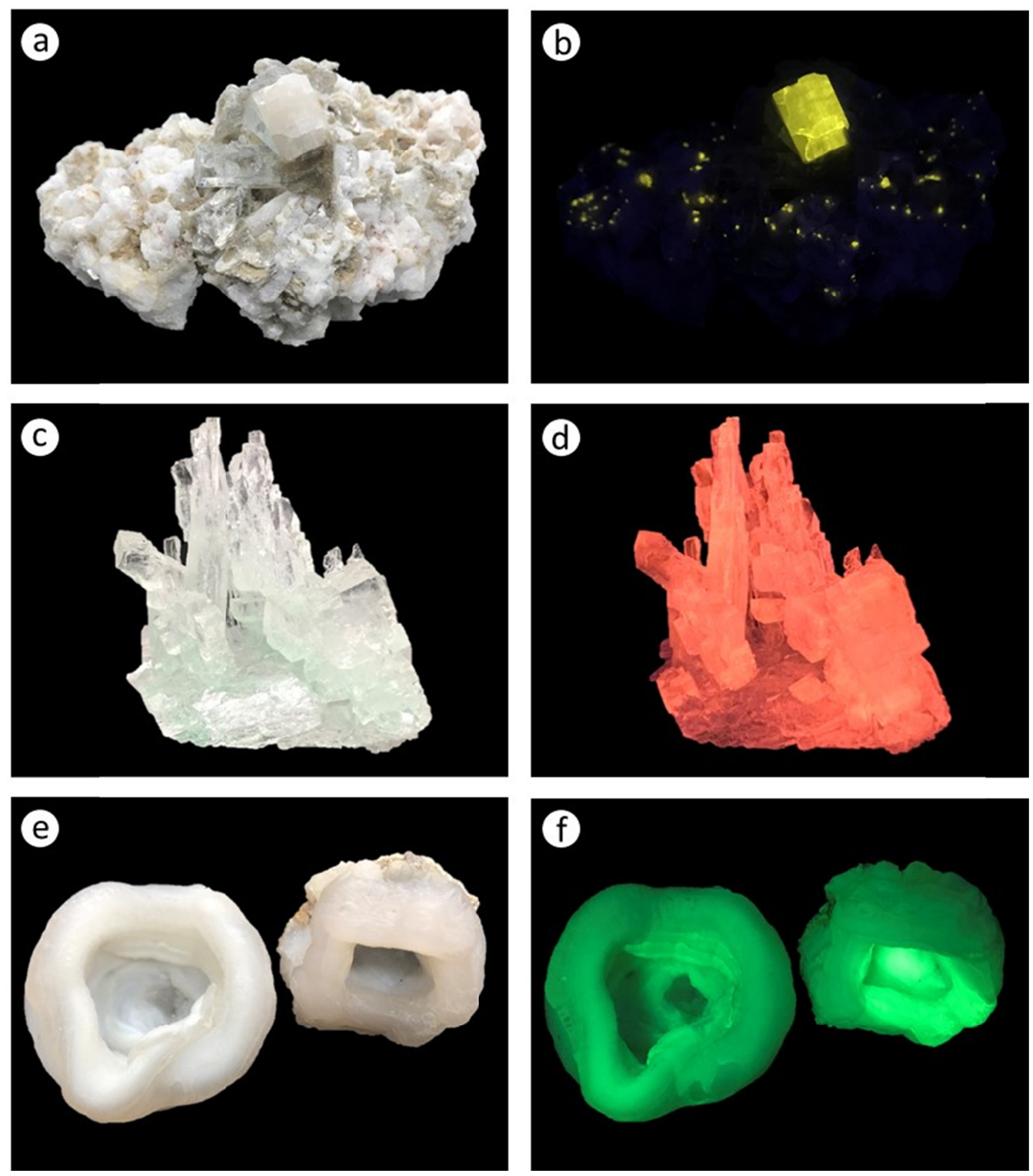

Figure 2. Minerals observed with natural light (left colum) and under UV light (right column). Only the name of the fluorescing minerals are reported. Where not indicated, the UV light source is shortwave (UVC). $a-b=$

Fluorapatite (Pakistan, Baltistan); c-d = Halite (Poland, Polkowice) ; e-f = Chalcedony (USA, New Mexico); g-h $=$ Aragonite (Italy, Sommatino; UVA); i-j Powellite (India, Nasik); k-1 = Scheelite (China Sichuan); $\mathrm{m}-\mathrm{n}=$ Scheelite (Austria, Salzburg); o-p = Calcite (China, Sichuan); $\mathrm{q}-\mathrm{r}=$ Calcite (in septaria, Madagascar); $\mathrm{s}-\mathrm{t}=$ Calcite and Willemite (USA, New Jersey); $\mathrm{u}-\mathrm{v}=$ Hardystonite, Clinohedrite, Willemite and Calcite (USA, New Jersey); $\mathrm{w}-\mathrm{x}=$ Calcite and Willemite (Zambia, Lusaka). 

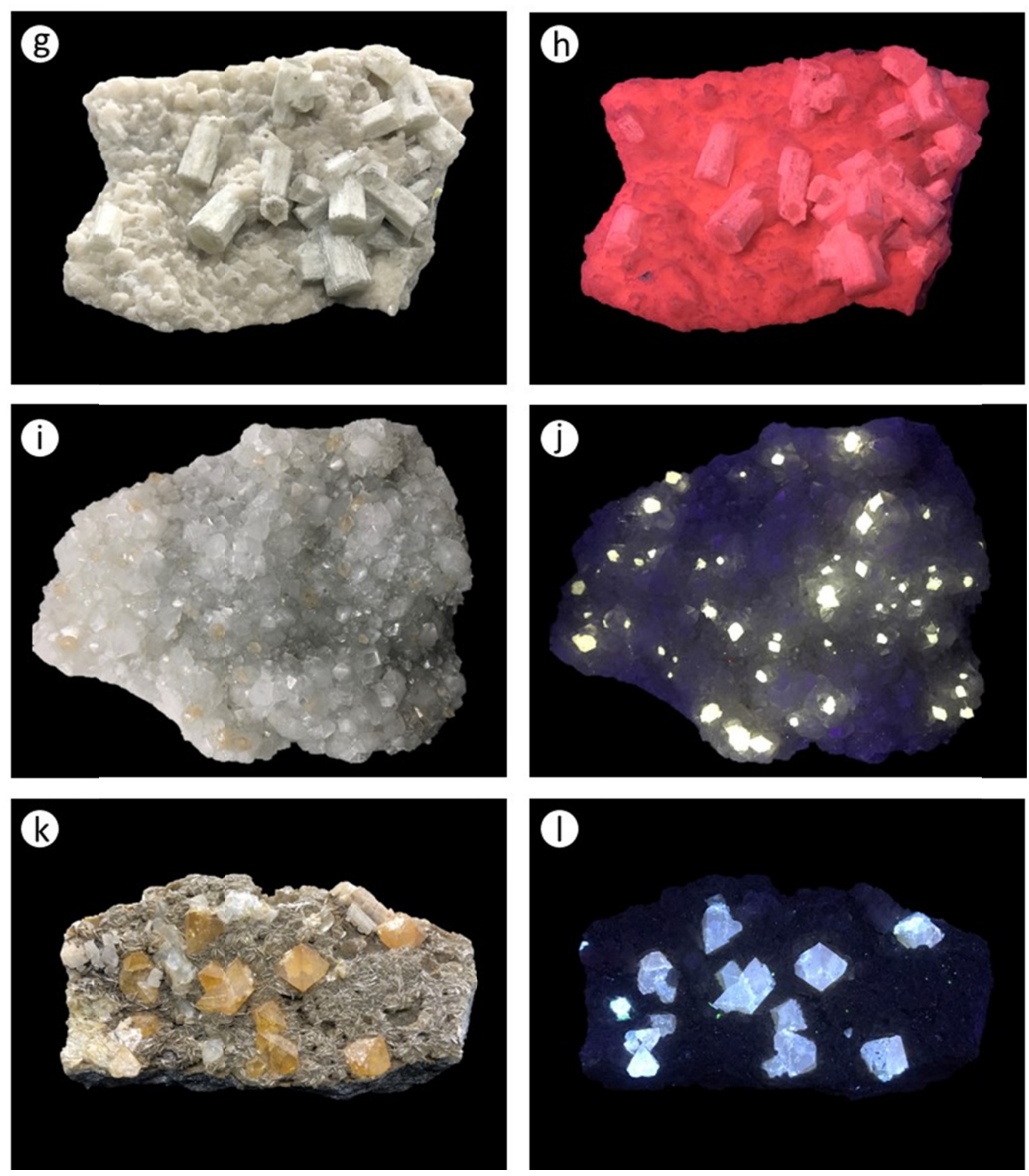

Figure 2. (continued). 



Figure 2. (continued). 

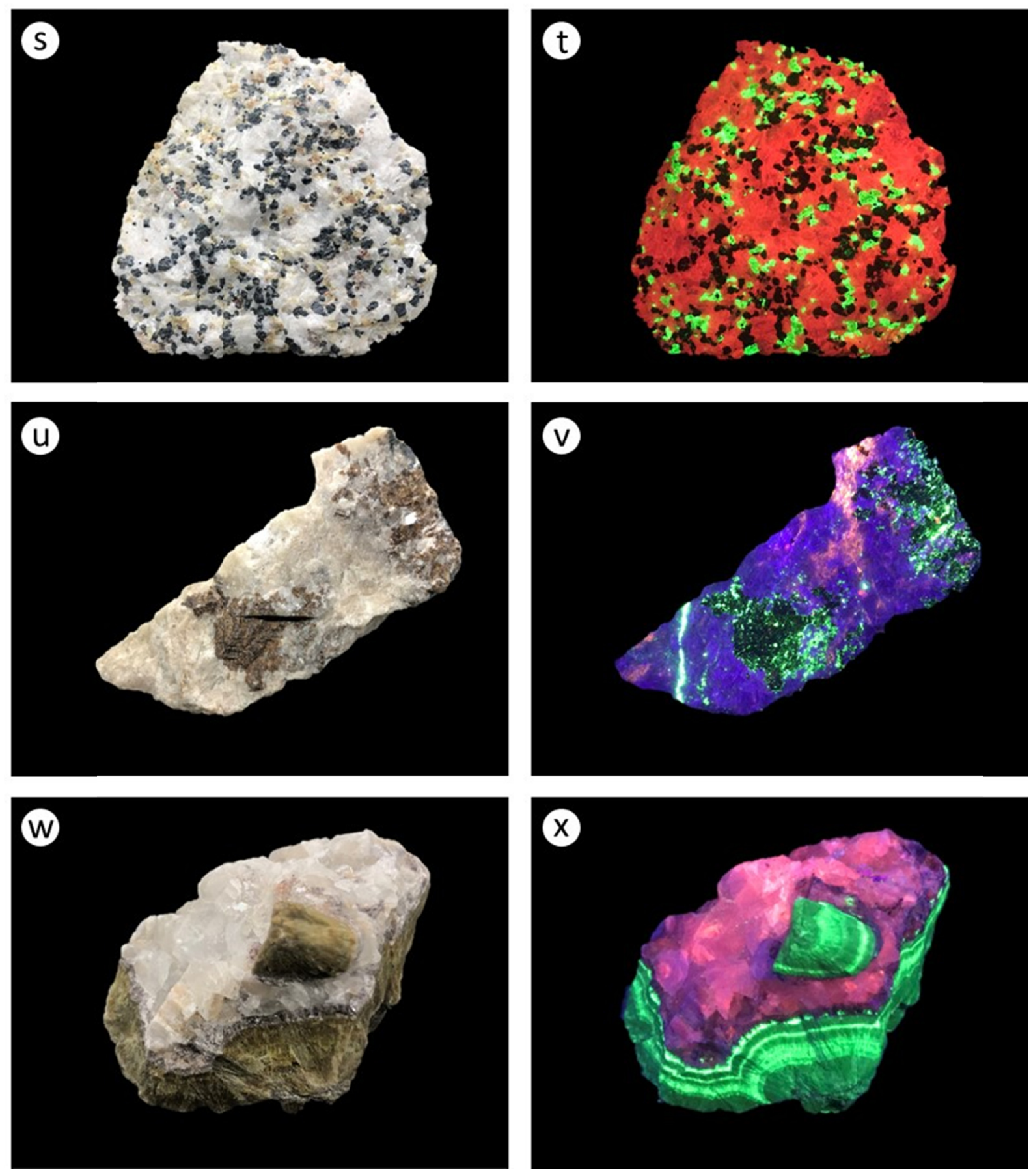

Figure 2. (continued). 
When an electron is hit by an energetic radiation (e.g., a photon with particularly high frequency), it can absorb the photon energy, being excited and moving to the outer shell (Figure 3). In this situation the electron is considered to be in an unstable excited state and soon will relax back to its original position (i.e., closer to the nucleus). When relaxing back to its ground state, the electrons of non-fluorescent minerals usually emit the entire excitation energy. It is more or less like considering a ball in a stadium. If we give energy to the ball (e.g., kicking it) it can reach the steps of the stadium. The distance the ball will reach depends on the energy input. The ball will stop to a given row (third, fifth or twelfth), but not in intermediate positions (i.e., it is impossible that it will stop in between the sixth and the seventh row of steps). In this case, kinetic energy (the kick) is transformed into potential energy. What happens to the electron "kicked off" by an energetic photon is analogous.

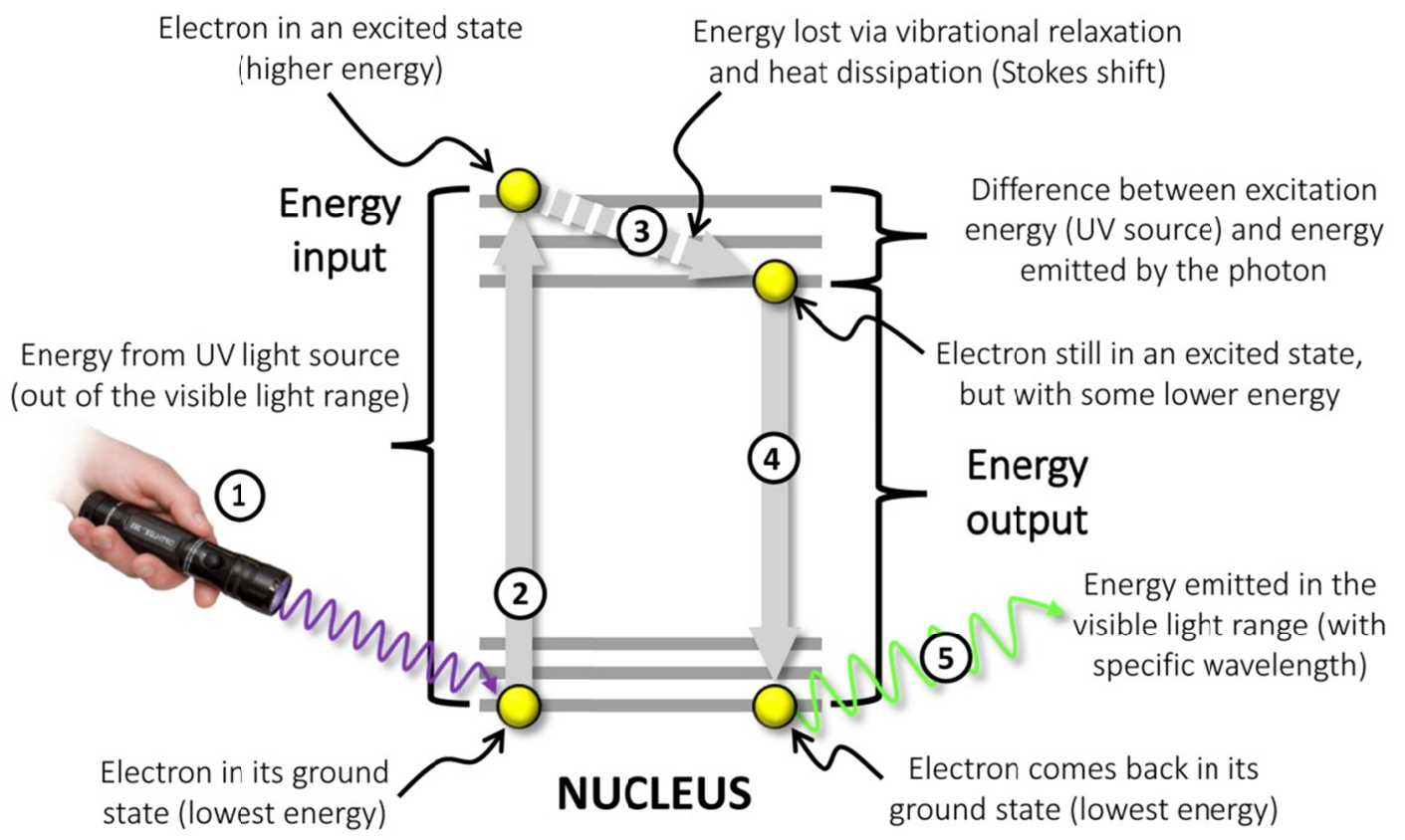

Figure 3. Explanation of fluorescence. (1) A source of high energy electromagnetic radiation (UV) hits the electron of specific elements in the crystal structure of a specific mineral or amorphous substance; (2) the electron is excited and jumps to higher energy orbital, adsorbing the input of energy; (3) the electron remains in its excited state, but loses some energy by vibrational relaxation and heat dissipation (the amount of this energy loss is called Stoke's shift); (4) the excited electron comes back in its ground state, emitting photons with lower energy; (5) the emitted photon has lower energy and, consequentially, lower frequency and longer wavelength, possibly entering in the narrow range of visible light. In this way, illuminated by an "invisible light", fluorescent minerals start emitting "visible light".

Basically, Stokes stated that the energy emitted must be less than the original energy absorbed. Lower energy is equivalent to lower frequency (eq. 2) and longer wavelength (eq. 1). This is graphically illustrated in Figure 3 . If the exciting photon belongs to UV radiation it is invisible to human eyes (i.e., a pure UVA light appears dark even if current is turned on and photons are emitted). This is obvious when dealing with x-ray tubes or, even more clearly, when observing $\gamma$-ray emitting radioactive minerals [e.g., uranium-bearing minerals such as uraninite $-\mathrm{UO}_{2}$ - or autunite- $\mathrm{Ca}\left(\mathrm{UO}_{2}\right)_{2}\left(\mathrm{PO}_{4}\right)_{2} 10 \mathrm{H}_{2} \mathrm{O}$ ]. Radioactive minerals do not glow but many of them emit electromagnetic wavelengths even shorter than $\mathrm{x}$-rays.

The discovery of x-rays in December 1895 was possible because Wilhelm Conrad Röntgen was attracted by the green fluorescence colour of barium platinocyanide-coated glowing screens when he turned on high voltage Crookes tube (e.g., Patton, 1993). The discovery of natural radioactivity, in the form of invisible rays able to expose photographic films, was possible because Antoine Henri Becquerel thought in 1896 that fluorescent uranium-bearing minerals had the capacity to emit the same rays recently discovered by Röntgen.Phosphorescence is related to the same process, but it takes longer for the excited electrons to relax back to their ground state. This means that when the UV light is turned off, it is possible that some mineral continues to emit light that can be perceived in the darkness. 


\section{The Origin of the Colours}

The origin of the colours depends on the capacity of the substances to absorb specific wavelengths of the visible light spectrum and reflect others. For example, when illuminated by sun light or white artificial light sources, a red T-shirt appears so to our eyes because the material it is made of absorbs all the wavelengths with the exception of those corresponding to red. A white T-shirt does not absorb any wavelength in the visible light spectrum, while a black tissue absorbs nearly all wavelengths. Window glass allows the transmission of the entire visible spectrum, while green plastic sheets absorb all the wavelengths with the exception of green. Experiments using normal light (e.g., white light bulbs or LEDs or solar light) easily illustrate this effect of absorption, transmission and reflection. UVA and UVB lamps can facilitate understanding.

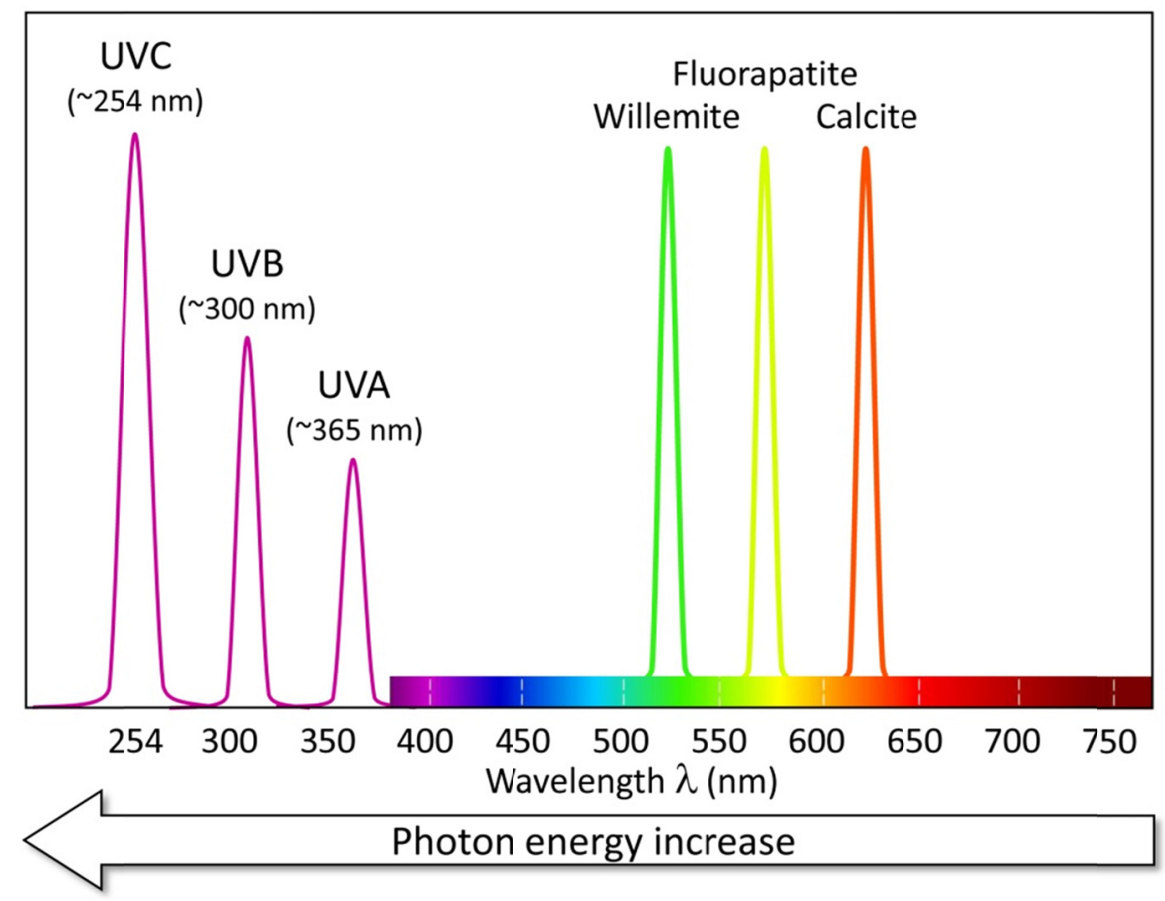

Figure 4. Examples of strongly focused UVA (LW), UVB (MW) and UVC (SW) hypothetical electromagnetic sources. The different height of the peaks is qualitatively indicative of the energy of photons. The three curves in the visible light range indicate the response to UVC light to willemite $\left(\mathrm{Zn}_{2} \mathrm{SiO}_{4} ;\right.$ Figs. $2 \mathrm{t}$ and $\left.2 \mathrm{x}\right)$, fluorapatite $\left[\mathrm{Ca}_{5}\left(\mathrm{PO}_{4}\right)_{3}\right.$ F; Fig. 2b] and calcite $\left(\mathrm{CaCO}_{3}\right.$; Figs. $2 \mathrm{p}$ and $\left.2 \mathrm{t}\right)$.

Some minerals give a fluorescent response only under UVA light, others only under UVC light and a few under both types of radiation, often with different fluorescent colours. If a piece of glass or any other transparent plastic material with limited thickness (i.e., $<1 \mathrm{~cm}$ ) is interposed in between an UVA light-activated fluorescent mineral and the UVA source nothing changes. UVA wavelengths are not substantially absorbed by glass or many plastic materials. This means that if a calcite has a UVA red fluorescent colour it continues to show the same colour. On the other hand, UVC wavelengths are easily absorbed even by thin (i.e., $1 \mathrm{~mm}$ ) transparent glass and plastic material. This means that a mineral showing brilliant fluorescent colour under UVC light loses its colour if a thin layer of glass or plastic sheet is interposed in between. In this way, the characteristics of some transparent materials to absorb specific wavelengths (e.g., $254 \mathrm{~nm}$ of UVC), and not others (e.g., $365 \mathrm{~nm}$ of UVA) can be easily demonstrated with surprising and easy experiments.

\section{Conclusions}

The use of inexpensive UVA lights or more expensive UVC lights has a large impact in activating curiosity to learn the origin of the colours as well as the principles of electromagnetic radiation. Compared to the typical aesthetic minerals shown in museums, fluorescent minerals cost less and, consequentially, are easily available (Figure 5). Fluorescence displays can be arranged using portable equipment (to be packed in a small case) and in any space, obscurity being the only necessary requirement. Wearing safety gloves and glasses is the only precaution, because a powerful UV light can burn eyes and skin (World Health Organization, 1994). 

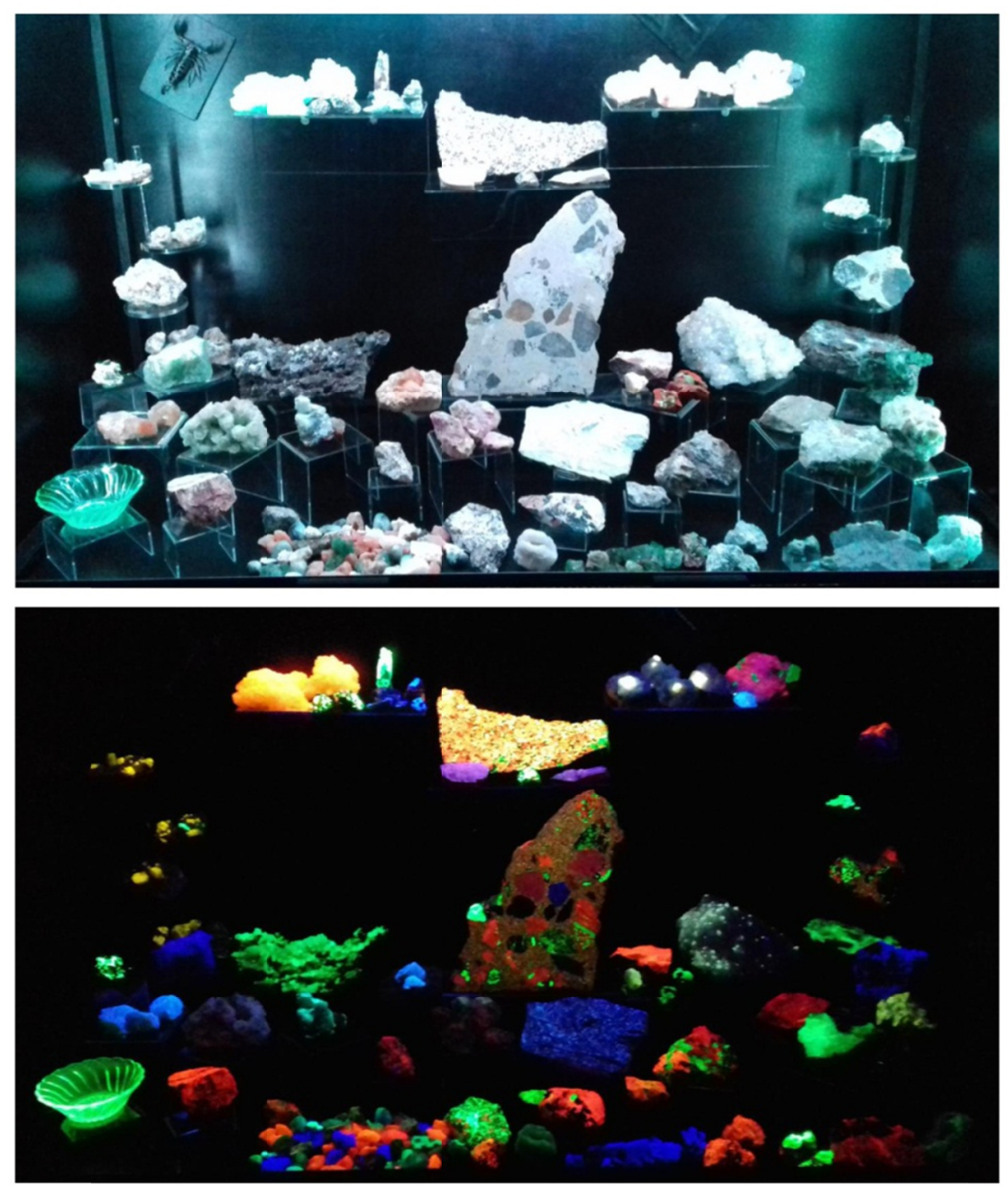

Figure 5. Fluorescent mineral showcase of MUST (Museo Universitario di Scienze della Terra - Earth Sciences

Museum of Sapienza University of Rome) with several fluorescent mineral and rock samples. (a) Samples observed with white artificial light; (b) the same samples observed with 144 W SW (UVC) light. The bowl in the lower left is made of uranium glass and shows the classical acid green fluorescent colour of minerals activated by the $\mathrm{UO}_{2}^{-2}$ ions. The scorpion in the top left of Fig. 5a is activated by LW (UVA) light (turned down in Fig. $5 b)$.

Concepts concerning the atomic structure, electron orbitals, the relation between energy and wavelength, the dual nature of the light, photons, electromagnetic spectrum, black-body radiation and even the origin of $\mathrm{x}$ - and $\gamma$-rays can be introduced using simple tools like UV light sources and common minerals.

Several simple experiments can be presented and discussed also with very young visitors. Among these some with the most emotional effects are reported below:

1) Prepare a display with black interior and a front transparent plastic cover, a normal LED strip (white light) and a UVC lamp inside, both fixed to the top. Additional UVA and/or UVB lamps can be placed inside and turned on in sequence or contemporaneously. When UV light is on, the white light has to be turned off. In this case, it is possible to show the visitors how the colours of fluorescent minerals are generated using different wavelengths (Fig. 5). At this step, the visitors can start reasoning about the different mechanisms of colours such as those generated by the visible (white) light (because of preferential absorption of specific wavelengths) and those generated by ultraviolet light (as consequence of the excitation of electrons coupled with some internal heating).

2) Less dangerous UVA flashlights can be used outside the case, allowing gloved visitors to handle UVA-reactive minerals (e.g., calcite, aragonite, scapolite, sphalerite, adamite, fluorite), animals (e.g., scorpions 
or chicken eggs) plants (e.g., mature robinia pseudoacacia stems) or seashells (e.g., leporicypraea mappa). It is special to see the visitors' reaction. Visitors can repeat the experiments buying cheap (less than 10-20 USD) UVA flashlights or Wood bulbs. The suggestion to search for fluorescent minerals in the dark nights is compelling for visitors.

3) Use UVA and UVC portable lamps to see the effect of Plexiglas (or normal glass) sheets in stopping or transmitting specific wavelengths. Repeat this experience with white light and coloured Plexiglas slabs to show the filter effect with visible light also.

4) Use phosphorescent paper on a wall illuminated by laser pointers with different wavelengths, e.g., red, green and blue, with increasing photon energy. Usually, the first two laser colours have a too little energy to cause phosphorescence on the paper, while blue (or violet) lasers can leave a track on the sheet. This helps the visitors to understand the difference of the intensity of the energy of different wavelengths, emphasizing that ultraviolet wavelengths are even more energetic.

5) Illuminate with UVA flashlight a mineral emitting fluorescent colours when illuminated by fluorescent UVA light (e.g., wernerite, calcite, ruby, noble spinel, aragonite, cerussite, fluorite, hauyne, hackmanite, and so on). Repeat the same procedure placing not expensive UV-blocking sunglasses in between the mineral and the UVA flashlight. Visitors wil realize that the UV wavelengths of the UVA flashlight are effectively neutralized and the reactive mineral stops emitting fluorescent light.

6) Use real banknotes and illuminate them with a normal flashlight and then with an UVA (or UVC) light source. Visitors will bet surprised in observing symbols of different colours invisible with normal light. If available, use also fake banknotes to show how distinguish real from counterfeit banknotes.

7) Use commercial sunscreen with high UV protection grade on fluorescent material (mineral, chicken egg shell, scorpion, wood, volcanic glass, seashell, fossil, uranium-glass) drawing a simple sign or a letter. Do the same with another substance (e.g., olive oil, toothpaste, honey) leaving a very thin layer on the fluorescent material. The signs of both UV-blocking sunscreen and other fluid subsances are invisible under white light, but only those drawn with sunscreen will result as dark areas when seen under UV lights, because sunscreen stops UV radiation and that part of the fluorescent material will not be excited. The areas covered by thin films of other fluid substances will fluoresce, because they do not stop UV radiation.

8) Use a fluorescent mineral with uranyl ion [e.g., autunite; $\mathrm{Ca}\left(\mathrm{UO}_{2}\right)_{2}\left(\mathrm{PO}_{4}\right)_{2} * 12\left(\mathrm{H}_{2} \mathrm{O}\right)$ ] to show the fluorescence colour (from bright green to lime green for autunite under both UVA and UVC light). Then use a Geiger counter to show the different kind of ionizing radiations emitted from a uranium-bearing substance. Measure the number of ionizing radiation of the radioactive substance placing the Geiger tube at a distance of 1-2 $\mathrm{cm}$ from the radioactive substance. In case of radioactivity caused by natural uranium, three types of ionizing radiations are always present. The first is associated to the emission of alpha $(\alpha)$ particles, consisting of ${ }^{4} \mathrm{He}$ nuclei, with two protons and two neutrons. This relatively heavy particle can travel only over a short distance from the source (no more than a few $\mathrm{cm}$ ) before being neutralized. It is easily blocked by a placing a thin sheet of paper in between the Geiger tube and the radioactive source. The difference in the number of counts with and without the sheet of paper measures the $\alpha$ particle flux. The second ionizing radiation is related to the emission of beta ( $\beta$ ) particles, nearly always consisting of electrons $\left(\beta^{-}\right)$generated when a neutron of the radioactive element is transformed into a proton, together with a neutrino to balance energy. Being nearly eigth thousand times less massive than $\alpha$ particles, $\beta^{-}$particles can travel longer distances, up to a couple of $\mathrm{dm}$. This radiation flux is not blocked by a sheet of paper, but by thicker metal slab, e.e., a $\sim 2-3 \mathrm{~mm}$ thick aluminium lid. Also in this case, the difference between the counts on the Geiger tube with and without the Al slab (and with or without the sheet of paper) can measure the flux of $\beta^{-}$or $\beta^{-}+\alpha$ particles. All the radiation coming out from the radioactive substance (i.e., autunite) shielded by an $\mathrm{Al}$ slab is in the form of the third ionizing radiation, known as the $\gamma$ rays. These are strongly energetic electromagnetic radiations, i.e., of the same family of UV light, but much more energetic. It is possible to show the public how our eyes cannot see the radiation emitted in the $\gamma$ ray range, similarly to the $x$ ray, microwave, radar and radio wave ranges. Instead of using natural radioactive U-bearing minerals, it is possible to use specific radioactive sources emitting $\alpha$ particles only $\left({ }^{210} \mathrm{Po}\right), \beta$ particles only $\left({ }^{14} \mathrm{C}\right)$ or $\gamma$ rays only $\left({ }^{133} \mathrm{Ba}\right)$.

9) To illustrate the difference between polychromatic and monochromatic lights, and the generation of the colours in everyday substances (jackets, t-shirts, book covers, fruit, and so on) show them to the public with artificial white light (e.g., that coming from cold-light LEDs). Then turn off it and turn on a low-pressure sodium vapour light (such as those used in old street lamplights; SOX bulbs). Actually, this kind of light reaches its maximum emission about 10-15 minutes after ignition, so it would be necessary to turn on it in advance. 
Low-pressure sodium lights effectively emit nearly monochromatic light peaking at $\sim 600 \mathrm{~nm}$ wavelength. When illuminated by this kind of light, all the other colours simply disappear, replaced by different shades of grey. This offers the possibility to show to the visitors how the colours of given substances (e.g., the green of a leaf or the blue of a tissue) are not intrinsic features, but are simply the wavelengths not absorbed and bounced back towards human eyes. A substance with a blue colour under sun light absorbs all the wavelegths with the exception of those corresponding to blue. If the same substance is illuminated with a light without the wavelength corresponding to blue (i.e., by using a low-pressure sodium light), it will result as black to dark gray. With this easy experiment, it is possible to illustrate the difference of "normal" colours and the fluorescent colours of the subsances.

To conclude, the experiments with light and, in particular, with fluorescent minerals, to be easily carried out in presence of variegated public, are numerous and very easy to perform. When exposed to fluorescent minerals for the first time, the people are blown away by it and go on to develop it as a hobby. This approach can foster a lifelong continued interest in science.

\section{Acknowledgements}

Thanks to Mark Cole, Axel Emmerman, Howard Green, Michael Isaacson and Conrad North for the help in sharpening the English style and in clarifying the basic concepts related to the luminescence of the minerals and the use of UV light sources. The continuous stimulus of the Fluorescent Mineral Society members is greatly appreciated. Warm thanks go also to Leandro Sala and Vincenzo Nasti of Gruppo Mineralogico Romano and to Guido Mazzoleni and Antonello Dallegno of Laser-induced PL Lab - Centro Studi Geominerario "Luciano Holzner" for the introduction to the magic world of fluorescent minerals. Thanks also to Roberto Appiani for his ability to take excellent pictures of fluorescent minerals, to Harald Stehlik for discussing about fluorescent minerals and to Sandra Biagetti, Alvaro Macchioni, Michele Macrì, Flora Panzarino, Catia Pietracatella and Linda Riti of Museo Universitario di Scienze della Terra for the continuous support in preparing and performing fluorescent mineral shows. The tens of students enrolled in Natural Sciences, Environmental Sciences and Geological Sciences courses of Sapienza University of Rome that have participated to this exciting experience has been strongly supportive. Special thanks go to Maurizio Varoli for having always believed in the potential of fluorescent minerals to move emotional feelings. The entusiasm of the thousands of visitors of MUST and of the Bologna and Rome mineral shows has been the fuel to improve day by day the scientific, didactic and emotional content of this approach. Ths article is dedicated to all of them, to the future visitors and all the curious people. Last but not least thanks to Enrica, Bianca and Laura for the patience in having a fluorescent mineral fanatic at home.

\section{References}

Herschel, W. (1800). Experiments on the refrangibility of the visible rays of the sun. Phyl. Trans. Roy. Soc., 90, 284-292. https://doi.org/10.1098/rstl.1800.0015

Mazzoleni, G., \& Appiani, R. (2010). Luminescence in mineral kingdom (Sandit Ed., p. 240).

Patton, D. (1993). Roentgen and the new light-Roentgen's moment. Part 3. The genealogy of Roentgen's $\begin{array}{llll}\text { barium platynocianide screen. Invest. } & \text { Radiol., } & \text { 28, }\end{array}$ https://doi.org/10.1097/00004424-199310000-00018

Stokes, G. G. (1852). On the change of refrangibility of light. Phil. Trans. Royal Soc., 142, 463-562. https://doi.org/10.1098/rstl.1852.0022

World Health Organization (1994). Ultraviolet radiation, p. 241. https://apps.who.int/iris/handle/10665/39901

\section{Declarations}

No original data are presented in this manuscript and no competing interests are present. Data sharing not applicable to this article as no datasets were generated or analysed during the current study. No funds have to be acknowledged, and I am the only one author of this contribution.

\section{Copyrights}

Copyright for this article is retained by the author, with first publication rights granted to the journal.

This is an open-access article distributed under the terms and conditions of the Creative Commons Attribution license (http://creativecommons.org/licenses/by/4.0/). 